\title{
Die Predigt aus soziologischer Sicht
}

Neben der Linguistik haben auch die Religionssoziologie und die Wissenssoziologie religiöse und spirituelle Settings in den Blick genommen. Obwohl an vielen Stellen christlich orientiert, setzt sich die Religionssoziologie ganz grundsätzlich mit allem auseinander, was unter dem schillernden Begriff der ,Religion * gefasst werden kann (vgl. Knoblauch 1999) und fragt religionenübergreifend nach „den Sozialformen der Religionen“ (Ludwig/Heiser 2014:1) v. a. unter den Aspekten ,der religiösen Erfahrung, dem religiösen Handeln, der religiösen Interaktion und der Bedeutung von Religion für die Gesellschaft insgesamt" (Ludwig/Heiser 2014:1). Es geht also grundsätzlich um die Frage nach den Erscheinungsformen von Religion als sozialem Phänomen sowie religiösen Handlungen und Institutionen (vgl. Heiser 2018:8). In diesem Zusammenhang werden Aspekte wie die gesellschaftliche Relevanz religiöser Praktiken oder der historisch und kulturell veränderbare Stellenwert religiöser (institutionalisierter) Rituale aus synchroner und diachroner Perspektive betrachtet (vgl. Heiser 2018). Das Untersuchungsfeld der Religion wird dabei auf unterschiedlichen Abstraktionsstufen perspektiviert. Ludwig/Heiser differenzieren zwischen der Makroebene, bei der z. B. die Aspekte der Legitimität, (Post)Säkularisierung und Rationalisierung im Fokus stehen (vgl. Ludwig/Heiser 2014:3), der Mikroebene mit den Themen der Werteorientierung, der Untersuchung (individueller) religiöser Praktiken etc. (vgl. Ludwig/Heiser 2014:4) und schließlich der Mesoebene, bei der es um Vergemeinschaftung, die Bildung von Organisationen und Netzwerken sowie um die „religiöse Vermarktlichung“ (Ludwig/Heiser 2014:7; siehe auch Heiser 2018) und Eventisierung geht. Die Autoren zeigen damit die Breite der religionssoziologischen Forschungsfelder und Analyseebenen auf. Im Zuge der Legitimierung religionssoziologischer Forschung wurde und wird nicht nur dafür plädiert eine breite Analyseperspektive einzunehmen und eine Vielzahl von Forschungsthemen zu berücksichtigen, sondern auch den Gegenstand der ,Religion“ selbst 
nicht auf institutionell spezialisierte, kirchlich orientierte Religion zu beschränken, sondern alle Spielarten von Religion als sozialem Phänomen in den Blick zu nehmen (vgl. Berger/Luckmann 2020:27). Entscheidend ist ein konstruktivistischer Zugang, für den sich u. a. Berger/Luckmann und Knoblauch aussprechen. Zentral ist dabei, das Alltagswissen über Religion als Ausgangspunkt wissenschaftlicher Untersuchung zu nehmen und danach zu fragen, was ethnokategorial als Religion, Glaube etc. konstruiert wird (vgl. Knoblauch 1999). Auch religiöse Ereignisse und die Frömmigkeitspraktiken unterschiedlicher Glaubensgemeinschaften werden dann entsprechend als soziale Konstruktionen verstanden (vgl. Knoblauch 1999). Gefragt wird etwa nach Formen der Transzendenzerfahrung (vgl. Schnettler 2011; Knoblauch/Soeffner 1999). Ulmer (1988) untersucht in diesem Zusammenhang die strukturelle Gestaltung von Konversionserzählungen und arbeitet die konstitutiven Elemente dieser kommunikativen Gattung heraus (siehe dazu auch Müller 2020). Schnettler (2011) untersucht mithilfe narrativer Interviews und Videoaufnahmen ,außeralltägliche visionäre Erfahrungen“ (2011:180) innerhalb der spirituellen Gemeinschaft Fiat Lux als performative Ereignisse. In seiner Studie identifiziert er sprachlich-stilistische sowie multimodale Besonderheiten, die eine visionäre Polyphonie kontextualisieren. Im katholischen Umfeld verortet sind wiederum die Studien von Knoblauch/Schnettler (2015) zu Marienerscheinungen. Auf der Grundlage von Videoaufzeichnungen arbeiten sie u. a. anhand interaktiver Ressourcen wie Mimik, Blick, Körperpositionierung und -orientierung heraus, wie die Transzendenzerfahrung einer Marienerscheinung von den ,betroffenen' Personen kontextualisiert und wahrnehmbar gemacht wird (vgl. Knoblauch/Schnettler 2015:644 ff). Zudem sind zahlreiche Untersuchungen zu unterschiedlichen Formen von Sepulkralkultur vorhanden. Benkel (2020) etwa analysiert Übergangsrituale im Zusammenhang mit Trauer und Tod. Gegenstand seiner Analyse sind sprachlich-semiotische Aspekte auf Grabsteinen. Im Bereich zwischen institutioneller Ritualität und individueller Frömmigkeitspraxis stehen religiöse Kleingruppen, die von Müller (2020) untersucht werden. Gerade in charismatischen Bewegungen bilden sie einen festen Bestandteil der Gemeindestruktur, bieten jedoch auch Raum für jeweils gruppenspezifische Abläufe und den Austausch subjektiver (religiöser) Erfahrungen sowie biblisch-religiösen Wissens. Dazu gehört, so Müller, auch die Diskussion der gottesdienstlichen Predigt (vgl. Müller 2020:308).

Die Studien von Schnettler (2011) und Knoblauch/Schnettler (2015) verdeutlichen zudem eine notwendige Hinwendung zur empirischen Religionsforschung und der Relevanz von audiovisuellen Aufnahmen für die ethnographische Forschung. Durch die Verschiebung des Erkenntnisinteresses hin zur „Analyse von Religion in ihrem ,natürlich vorkommenden Kontext““ (Schnettler 2011:169) und 
der „möglichst umfassende[n] und genaue[n] Rekonstruktion religiöser Phänomene" (Schnettler 2011:169) zeigt sich, dass die Auswertung von schriftlich vorliegenden Dokumenten wie Predigtmanuskripten, Gedächtnisprotokollen oder Feldnotizen nicht mehr ausreicht. Neue erhebungstechnische und methodische Verfahren werden notwendig (siehe Kapitel 11 und 12).

Diese vorgestellten religionssoziologischen Studien setzen sich explizit mit Formen religiösen Wissens auseinander und der Frage, ,in welchen Formen religiöses und weltanschauliches Wissen kommuniziert wird" (Schnettler et al. 2020:3). Damit bewegen sich die Untersuchungen an der Grenze zur modernen Wissenssoziologie, die es sich zur Aufgabe macht die ,gesellschaftlichen Formen des Wissens" (Berger/Luckmann 2020:28), gerade auch des religiösen Wissens, und die damit verbundene gesellschaftliche Konstruktion von Wirklichkeit zu untersuchen (vgl. Schnettler et al. 2020:5). Entscheidend ist für die an Religion interessierte Wissenssoziologie die Ebene von Sprache und Kommunikation. Religion, religiöses Wissen bzw. allgemeiner Weltanschauungen werden nicht als feste Entitäten, sondern als ,gesellschaftlich und kommunikativ konstruiertes Phänomen" (Schnettler et al. 2020:6) konzeptualisiert. Ein zentrales Modell der Wissenssoziologie ist in diesem Zusammenhang das Konzept der kommunikativen Gattungen (siehe Kapitel 8). Auffällig ist jedoch, dass es sowohl in der Religionssoziologie als auch in der Wissenssoziologie keine umfassenden Untersuchungen christlicher Verkündigung face-to-face, d. h. der christlichen Predigt, gibt. Dies mag auch an der Ausweitung des Religionsbegriffs und der damit verbundenen Abwendung von christlichen, institutionalisierten Formen der Verkündigung liegen. Während sich Max Weber noch mit der Predigt als „Kollektivbelehrung über religiöse und ethische Dinge“ (Weber 1956:283) im Zuge religiöser Vergemeinschaftung auseinandersetzt, fragt die moderne (Religions)Soziologie weniger nach der Predigt als eigenständigem Ereignis, sondern nach Religion, Kirche und Gottesdienst als Institution und Organisation, die vor allem der Bewältigung von Kontingenz dient (vgl. Kern/Pruisken 2017:408). In diesem Zusammenhang wird dann auch die Predigt als Form der Kontingenzbewältigung etabliert, d. h. mit, durch und in der Predigt geht es darum, ,dem Leben trotz aller Zufälligkeit und Unglücksfälle einen Sinn abzugewinnen“ (Ebert 2017:317). Wie dies jedoch im Einzelnen geschieht, bleibt unklar. Einzig in den Anfängen der modernen Wissenssoziologie richtete sich die Forschung in Ansätzen auf den Gegenstand Predigt. Jedoch liegen auch hier keine Studien vor, die sich explizit mit christlichen, gottesdienstlichen Predigten auseinandersetzen. Luckmann etwa betrachtet den Übergang von der Predigt, als mehr oder weniger exklusiv dem kirchlich-religiösen und institutionellen Bereich zugeordneter kommunikativer Gattung, hin zur säkularen, alltagsweltlichen und alltagssprachlichen 
Gattung der Moralpredigt (vgl. Luckmann 1986, 1998, 1999a und 1999b, 2001). Er definiert die gottesdienstliche Predigt in diesem Zusammenhang als ,historische, kirchlich-institutionell bestimmte kommunikative Gattung“ (Luckmann 1999a:43 f.) sowie als ,nicht-rekonstruktive[ ] (didaktische[ ], erbauliche[ ])“ Gattung (Luckmann 1997:14) und grenzt davon rekonstruktive Gattungen (Konversionserzählungen, Interviews, Klatsch) ab (vgl. Luckmann 1997:14 f.). Zudem bestimmt er sie als moralische Gattung und benennt auf der Grundlage der theologischen Entwicklungsgeschichte die ,moralische Unterweisung und Ermahnung“ (vgl. Luckmann 1999a:45) als eine zentrale Funktion. Inwiefern diese Einteilung zutrifft und worin sie sich begründet, wird sowohl bei Luckmann als auch in der sich daran anschließenden weiteren Gattungsforschung nicht überprüft. Vielmehr zeigt Luckmann selbst auf, dass es die Religions- und Wissenssoziologie schuldig geblieben sind, die kirchliche Predigt synchron wie diachron systematisch hinsichtlich ihrer Formen und Funktionen sowie ihrer „Bedeutung für die Vermittlung wesentlicher Bestandteile eines gesellschaftlichen Wissensvorrats und einer verpflichtenden moralischen Ordnung über viele Jahrhunderte" (Luckmann 1998:394) zu analysieren. Luckmann betont in seiner Untersuchung, dass sich die formale Gestaltung von Predigt und Moralpredigt soweit unterscheiden, dass z. T. nur noch die Gattungsbezeichnung auf eine Verwandtschaft schließen lässt, und einzelne Rudimentelemente vorhanden sind. Was jedoch eine Predigt formal ausmacht, bleibt in seinen Untersuchungen stichwortartig verkürzt und ungenau. Möglicherweise hängt die Vernachlässigung der christlichen Predigt innerhalb der Forschung zu kommunikativen Gattungen mit einer grundsätzlichen Haltung zusammen, die die Predigt zwar als historisch höchst relevant und einflussreich verortet (vgl. Luckmann 2001:388 f.), ihre aktuelle Wirkmächtigkeit jedoch mit einigen Fragezeichen sieht:

Im kommunikativen Haushalt der meisten modernen Gesellschaften mit einer christlichen Vergangenheit [...] spielt die kirchliche Predigt eine kleine, im gesellschaftlichen Gesamtzusammenhang wahrscheinlich zu vernachlässigende Rolle. Der Kreis ihrer Adressaten schrumpft seit Generationen, evangelische und katholische Theologen verkünden, dass die Predigt in einer bösen Krise steckt. (Luckmann 1998:395 f; siehe dazu auch Luckmann 2001:389) ${ }^{1}$

Das von Luckmann aufgezeigte Forschungsdesiderat einer konzisen Gattungsbestimmung (synchron und diachron) besteht nach wie vor, denn alle sich mit der Gattungsanalyse auseinandersetzenden Arbeiten folgen seiner Einschätzung

\footnotetext{
${ }^{1}$ Diese ,Krise der Predigt ' wird auch in der Homiletik immer wieder zum Ausgangspunkt für Überlegungen und Studien über die (moderne) Predigt genommen (siehe Kapitel 6).
} 
der Predigt als prototypischer kommunikativer Gattung. Sie bleiben es schuldig, die Predigt nicht nur als Gattung zu bezeichnen, sondern sie auf der Grundlage empirischer Daten auch als solche auf den drei Ebenen des Gattungskonzepts (siehe Kapitel 8) zu beschreiben. Lediglich beispielhafte Erwähnung findet sie als komplexe monologische Gattung und wenn es um die Frage nach der Kontextgebundenheit und Reflexivität kommunikativer Gattungen geht (vgl. z. B. Schnettler 2011:170 f., Günthner 1995:209, Günthner 2018:40).

Wie dieses Verhältnis jedoch konkret aussieht, bleibt unklar. Als einzige Literaturreferenz verweist z. B. Knoblauch (2003:150) auf die Dissertation von Ayaß (1997a), in der sie das ,Wort zum Sonntag ' als mediale kommunikative Gattung hinsichtlich seiner Struktur, seiner kommunikativen Funktionen, den erkennbaren dialogischen Elementen und Aspekten moralischer Kommunikation untersucht (vgl. Ayaß 1997a,b und 1998). Dazu arbeitet sie mit einem Video-Korpus sowohl katholischer als auch evangelischer Sprecher und Sprecherinnen. Ayaß verweist deutlich darauf, dass es sich beim Wort zum Sonntag nicht nur um eine „mediale Kurzpredigt" (Ayaß 1997b:222) handelt, sondern um eine eigenständige kommunikative religiöse Gattung und „moderne Form christlicher Prophetie“ (Ayaß 1997b:222), die als religiöse Ansprache „Fragen der christlichen Lebensführung“ (Ayaß 1997b:223) zum Gegenstand hat. Die Unterschiede zu einer gottesdienstlichen Predigt scheinen hier schon auf. Besonders deutlich wird dies in der Struktur der Gattung Wort zum Sonntag, die Ayaß als , heiligen Bogen' bezeichnet (vgl. Ayaß 1997b:229-231): meist allgemeiner Einstieg ohne christlichen Bezug, (negative) „Schilderung menschlichen Daseins“ (Ayaß 1997b:229) als ,irdisches Jammertal“ (Аyaß 1997b:229), der Übergang „vom Dunkel ins Licht“ (1997b:230) durch dezidierte Bezüge zum Christentum (z. B. Bibelzitate, Rekonstruktion biblischer Erzählungen etc.) und schließlich ein moralisches Ende. Wie die nachfolgenden Analysen zeigen werden, lässt sich für die christliche Predigt - und speziell den Wortbeitrag - unter ko-präsenten Beteiligten innerhalb eines Gottesdienstes kein solcher prototypischer inhaltlicher Ablauf erkennen. Auch wenn sich auf der Ebene des Rezipientenzuschnitts, der Dialogizität und dem Umgang mit biblischen Texten Parallelen und Ähnlichkeiten zwischen dem Wort zum Sonntag und einer Predigt zeigen (siehe Kapitel 17), lassen sich diese beiden Verkündigungsformate strukturell also nur bedingt miteinander vergleichen. So weist das Wort zum Sonntag als Medienformat in seiner Positionierung innerhalb eines inhaltlich wie ästhetisch höchst heterogenen massenmedialen Programmkontextes deutliche Unterschiede hinsichtlich der Rezipierenden, der Konzeption, der Ästhetik, des moralischen Gehalts und der Formen der Wissenszuschreibung und Wissensvermittlung im Vergleich zu einer Sonntagspredigt auf (siehe Kapitel 16). Es greift also deutlich zu kurz, von der kommunikativen Gattung des 
Wortes zum Sonntag auf die kommunikative Gattung der christlichen (Sonntags)Predigt zu schließen. Eine Akzentuierung des soziologisch-linguistischen Gattungsbegriffs an der Predigt und eine Beschreibung der typischen und spezifischen Merkmale der christlichen, gottesdienstlichen Predigt anhand natürlicher Audio- und Videodaten ist ein Forschungsdesiderat, das durch die vorliegende Untersuchung bearbeitet wird.

Open Access Dieses Kapitel wird unter der Creative Commons Namensnennung 4.0 International Lizenz (http://creativecommons.org/licenses/by/4.0/deed.de) veröffentlicht, welche die Nutzung, Vervielfältigung, Bearbeitung, Verbreitung und Wiedergabe in jeglichem Medium und Format erlaubt, sofern Sie den/die ursprünglichen Autor(en) und die Quelle ordnungsgemäß nennen, einen Link zur Creative Commons Lizenz beifügen und angeben, ob Änderungen vorgenommen wurden.

Die in diesem Kapitel enthaltenen Bilder und sonstiges Drittmaterial unterliegen ebenfalls der genannten Creative Commons Lizenz, sofern sich aus der Abbildungslegende nichts anderes ergibt. Sofern das betreffende Material nicht unter der genannten Creative Commons Lizenz steht und die betreffende Handlung nicht nach gesetzlichen Vorschriften erlaubt ist, ist für die oben aufgeführten Weiterverwendungen des Materials die Einwilligung des jeweiligen Rechteinhabers einzuholen. 